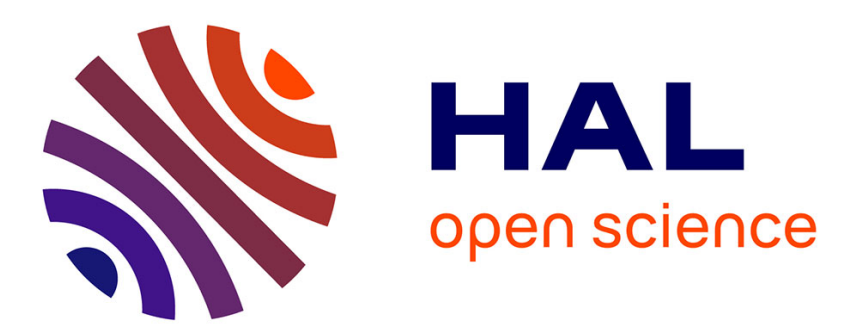

\title{
A robust ultrasound-based visual servoing approach for automatic guidance of a surgical instrument with in vivo experiments
}

\author{
Marie-Aude Vitrani, Guillaume Morel, Nicolas Bonnet, Mourad Karouia
}

\section{- To cite this version:}

Marie-Aude Vitrani, Guillaume Morel, Nicolas Bonnet, Mourad Karouia. A robust ultrasound-based visual servoing approach for automatic guidance of a surgical instrument with in vivo experiments. The First IEEE/RAS-EMBS International Conference on Biomedical Robotics and Biomechatronics, 2006. BioRob 2006., Feb 2006, Pisa, Italy. pp.35-40, 10.1109/BIOROB.2006.1639056 . hal-01170702

\section{HAL Id: hal-01170702 https://hal.sorbonne-universite.fr/hal-01170702}

Submitted on 2 Jul 2015

HAL is a multi-disciplinary open access archive for the deposit and dissemination of scientific research documents, whether they are published or not. The documents may come from teaching and research institutions in France or abroad, or from public or private research centers.
L'archive ouverte pluridisciplinaire HAL, est destinée au dépôt et à la diffusion de documents scientifiques de niveau recherche, publiés ou non, émanant des établissements d'enseignement et de recherche français ou étrangers, des laboratoires publics ou privés. 


\section{A robust ultrasound-based visual servoing approach for automatic guidance of a surgical instrument with in vivo experiments}

\author{
Marie-Aude Vitrani and Guillaume Morel \\ University Pierre and Marie Curie-Paris 6, CNRS(FRE2507) \\ Laboratoire de Robotique de Paris (LRP) \\ 18 route du panorama, 92265 Fontenay-aux-Roses, France \\ $\{$ vitrani, morel\}@ robot.jussieu.fr
}

\author{
Nicolas Bonnet and Mourad Karouia \\ University Pierre and Marie Curie-Paris 6 \\ Pitié-Salpêtrière Hospital, Cardiac Surgery Service \\ 47-83 boulevard de l'Hpital,75013 Paris, France
}

\begin{abstract}
This paper presents a visual servoing approach for automatic guidance of an instrument. In this approach, the visual sensor is an ultrasound probe that observes an instrument inserted inside the beating heart of a patient and manipulated by a robot. Following the preliminary results presented in [1], the present paper provides a new parameterization of the visual feature signal that allows for enhanced robustness, and shows the first in vivo results on this ongoing research.
\end{abstract}

Index Terms-Surgical robotics, ultrasound imaging, visual servoing.

\section{INTRODUCTION}

Ultrasound imaging systems are low cost, non invasive devices that provide high resolution images in real time. They are mainly used in the sole aim of producing images. However, they can also be employed for intervention procedures, where the surgeon moves an instrument inside a patient while observing the motion of the instrument with an outer ultrasound probe. This requires high skills for coordinating the hands holding the instrument and the probe. Indeed, the ultrasound image gives only a $2 \mathrm{D}$ cross-section of the 3D working volume which contains no depth information. Therefore, manual ultrasound guided interventions are limited to simple tasks, such as puncture using a needle [2].

Within this context, robotic systems are being developed in order to perform ultrasound image-based guidance in a more performing way than manual procedures. In the literature, the proposed systems can be divided into two groups.

In the first group, a robot is used to manipulate an ultrasound probe. In most of these papers the ultrasound image is considered as an output of the system, but it is not used as a measurement device to control the robot motion. Here, the robot allows for long distance telemanipulation for remote ultrasound imaging (e.g. [3], [4]), or for enhanced quality imaging thanks to the control of the force applied by the probe to the patient [5]. In contrast, in [6], a telemanipulated probe holder is presented with automatic visual tracking features. This system allows for enhanced imaging capabilities, such as physiological motion compensation. In [7], another visually servoed system is proposed, which allows for positioning a probe along 6 degrees of freedom, with respect to an artificial phantom with known geometry. This method is aimed at being applied to the calibration of a 3D ultrasound imaging system. In the second group of papers, which correspond to the scope of our research, a robot is used to move an instrument under the supervision of an ultrasound imaging system. In [8], a computer-assisted robotic system is used for ultrasoundguided biopsy. A robot holds a needle, which is to be inserted into an organ under the supervision of the surgeon. The system provides the surgeon with a reconstructed 3D view of the probe and the needle, but there is no automatic guidance feature. Furthermore, the 3D reconstruction requires a number of additional equipments to localize the ultrasound probe and the needle. In [9], a robotic system is presented, which includes a manipulator moving a surgical instrument toward a target. Automatic tracking of the target is provided, and fed to the robot controller. The visual servo loop is not expressed in the image; as a result, the system requires additional equipment to measure the position of the probe with respect to the manipulator, together with extensive calibration procedures to estimate the geometric transforms between several equipments. The system exhibits positioning errors as large as $15 \mathrm{~mm}$ RMS. In [10], direct visual servoing is used to automatically guide a needle for a percutaneous cholecystostomy. A mechatronic device is presented, comprising a ultrasound probe and a 2 degrees of freedom needle manipulator. The needle is mechanically constrained to lie within the ultrasound plane. Its orientation and penetration are automatically controlled thanks to a visual servoing loop aimed at placing the needle tip into a tracked target. Here, as the needle and the probe are mechanically connected, there is no need for any extra devices to locate the probe with respect to the needle manipulator. However, this research applies only for a particular kind of planar tasks. In [1], we have considered the broader problem of controlling an instrument that does not entirely lie in the ultrasound plane. The proposed approach is based on direct visual servoing. The present paper provides the most recent results of this research: the overall system design presented in [1] is kept (see Sect. II), but a new parameterization for the image features to be servoed is proposed (see Sect. III). With 
this parameterization, the closed loop system exhibits more robustness (see Sect. IV), and the control law does not require any knowledge of the probe location with respect to the robot. Also, the parameterization allows for coping with the visibility problem (see Sect. V). Finally, in vivo experiments are presented for the first time in Sect. VI.

\section{VISUALLY SERVOED SYSTEM OVERVIEW}

\section{A. Mitral valve surgery}

The mitral valve is one of the four cardiac valves, Fig. 1. It separates the left atrium and the left ventricle, and ensures that the blood flows from the atrium to the ventricle when the heart beats. It is made up of two leaflets attached inside the ventricle through chords. These chords help opening and closing the valve during the cardiac cycle. When a chord breaks it drastically affects the valve efficiency. The valve leaflets do not completely seal when the valve is closed so that the blood flows back into the atrium. This pathology is called mitral valve insufficiency. It is diagnosed with ultrasound images of the heart.

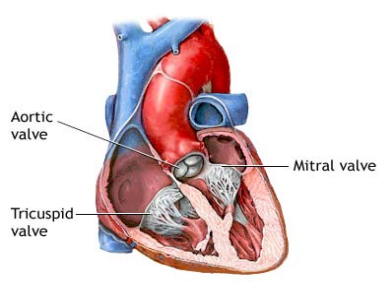

(a) cardiac valves

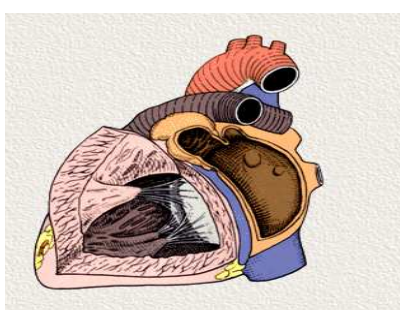

(b) left ventricle
Fig. 1. Mitral Valve

To repair the valve, a classical intervention consists in attaching a neo-chord in order to replace the broken chord. This operation requires the installation of a bypass machine. Then the atrium is open to reach the valve. Thus, this procedure is very invasive for the patient. The procedure proposed in this paper allows for beating heart surgery. The idea is that a pair of forceps is inserted inside the left ventricle through the heart tip. This instrument is moved towards the leaflet using ultrasound images. These images can be produced by a transoesophagus probe or by a probe placed in contact with the heart wall. One tip of the neo-chord is then attached to the leaflet and the other one outside the ventricle. This procedure cannot be performed manually because the coordination between the hands holding the probe and the instrument is too difficult. In the proposed robotic system, this coordination is realized automatically.

\section{B. System description}

The overall system is sketched in Fig. 2. It involves a robot holding an instrument, inserted into the heart through a trocar. The instrument is observed by an ultrasound probe, which images are sent in real time to a computer. In the chosen configuration, each jaw of the instrument intersects the ultrasound plane. Thus, two blobs are visible in the image, which allows for the instrument localization, thanks to appropriate image processing algorithms, see [11].

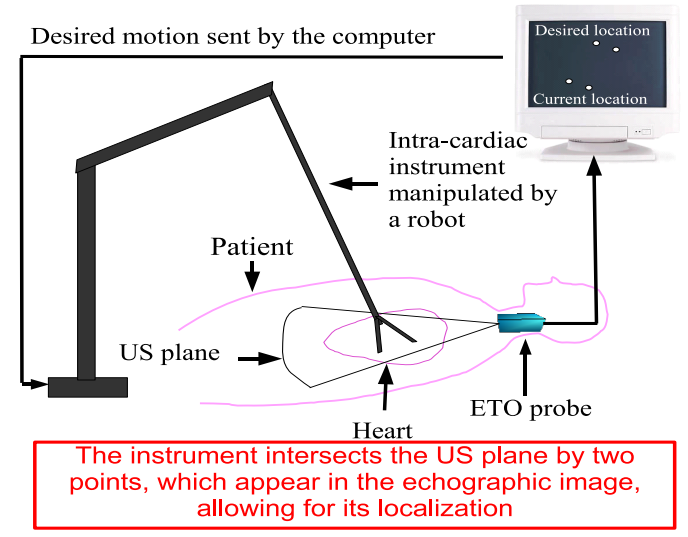

Fig. 2. System Description

More precisely, the image processing provides in real time the coordinates of the center of gravity $P_{1}$ and $P_{2}$ of the two blobs representing the instrument image. The goal of the controller is to move $P_{1}$ and $P_{2}$ at a desired location, which the surgeon specifies by simply clicking in the image. The controller is inspired from conventional visual servoing techniques, which principle is briefly recalled in the next.

\section{Visual servoing principle}

A visual servoing loop (see Fig. 3) is a controller aimed at moving a set of image features $\mathbf{s}$ extracted from the image towards a desired value $\mathbf{s}_{\mathbf{d}}$.

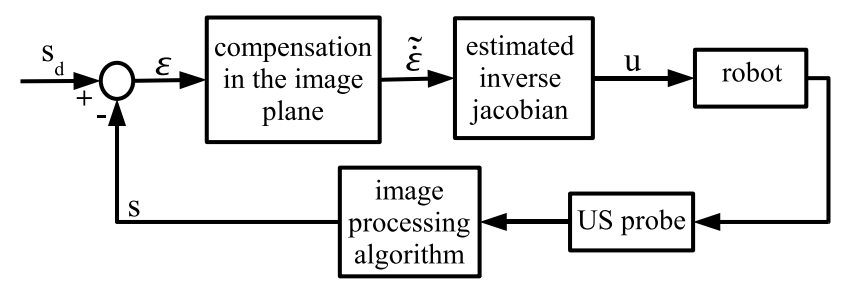

Fig. 3. Visual servoing loop principle

Most commonly, the command $\mathbf{u}$ sent to the robot is calculated with the following control law:

$$
\mathbf{u}=-\lambda \widehat{\mathbf{J}}_{\mathbf{s}}^{-1}\left(\mathbf{s}_{\mathbf{d}}-\mathbf{s}\right)=-\lambda \widehat{\mathbf{J}}_{\mathbf{s}}^{-\mathbf{1}} \boldsymbol{\varepsilon}
$$

where $\lambda$ is a scalar proportional gain and $\widehat{\mathbf{J}}_{\mathbf{s}}$ is the estimate of the image jacobian matrix $\mathbf{J}_{\mathbf{s}}$ defined by:

$$
\dot{\mathbf{s}}=\mathbf{J}_{\mathbf{s}} \mathbf{u}
$$

The associated closed loop behavior is then:

$$
\dot{\varepsilon}=-\dot{\mathbf{s}}=-\lambda \mathbf{J}_{\mathbf{s}} \widehat{\mathbf{J}}_{\mathbf{s}}^{-1} \varepsilon
$$

A sufficient condition for the stability is that the matrix product $\mathbf{J}_{\mathbf{s}} \widehat{\mathbf{J}}_{\mathbf{s}}^{-1}$ is positive definite. In this case, the final error is guaranteed to be null. 
It can be seen from (3) that the robustness of the control law strongly depends on both the structure of $\mathbf{J}_{\mathbf{S}}$ and the faculty to accurately estimate $\mathbf{J}_{\mathbf{s}}$. In other words, an important part of the design of a visual servo loop is the choice of the features in the image $\mathbf{s}$ and the computation of both $\mathbf{J}_{\mathbf{s}}$ and its estimate, [12].

A first set of features was presented in [1]. The feature vector $\mathrm{s}$ was simply constructed from grouping the coordinates of $P_{1}$ and $P_{2}$. In this paper, another set of features is proposed which results in a more robust control law.

\section{FEATURES AND IMAGE JACOBIAN}

\section{A. Image features}

The studied features are represented on Fig. 4(a). The feature vector $\mathbf{s}$ is chosen as:

$$
\mathbf{s}=\left[\begin{array}{llll}
{ }^{S} x_{P} & \delta & { }^{S} y_{P} & \theta
\end{array}\right]^{T}
$$

where:

- $\left[{ }^{S} x_{P},{ }^{S} y_{P}\right]$ are the image coordinates of the point $P$ defined as the midpoint of $P_{1}$ and $P_{2}$,

- $\delta$ is the distance between $P_{1}$ and $P_{2}$,

- $\theta$ is the angle between the image horizontal axis and $\mathbf{d}_{\mathbf{P}_{1} \mathbf{P}_{2}}$.

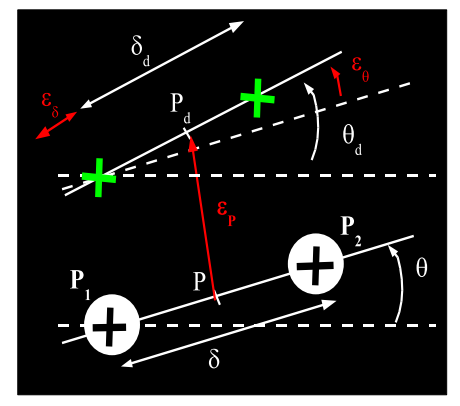

(a) Features in the image

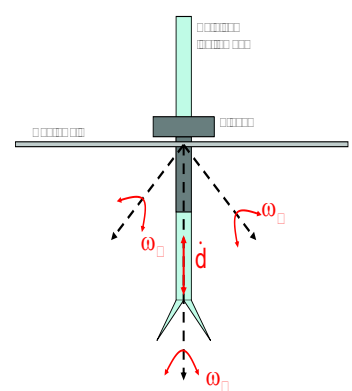

(b) Command vector
Fig. 4. Features and command

From this choice, a crucial issue is to provide a kinematic model as given in (2).

\section{B. Geometrical modeling}

In order to derive a model for the control design, a first modelling simplification consists in assuming that $P_{1}$ and $P_{2}$ coincide with the two points $M_{1}$ and $M_{2}$ that correspond to the intersection of two lines (representing the instrument jaws) and a plane $\Pi$ (representing the ultrasound probe, which is assumed to provide a $2 \mathrm{D}$ cross-section of the $3 \mathrm{D}$ workspace).

In the next, four orthonormal coordinate frames will be used, Fig. 5(a):

- $\mathcal{F}_{T}=\left\{T ; \mathbf{i}_{T}, \mathbf{j}_{T}, \mathbf{k}_{T}\right\}$ is the coordinate frame attached to the robot base at point $T$ which denotes the trocar center (fulcrum point),

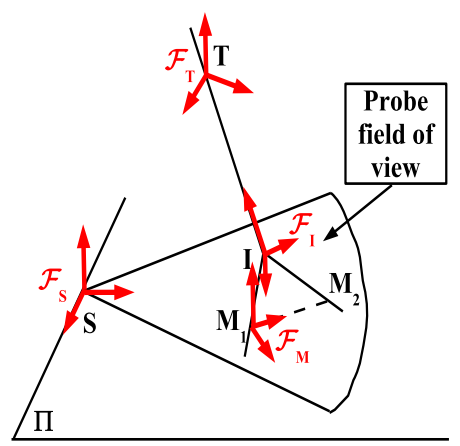

(a) Frame description

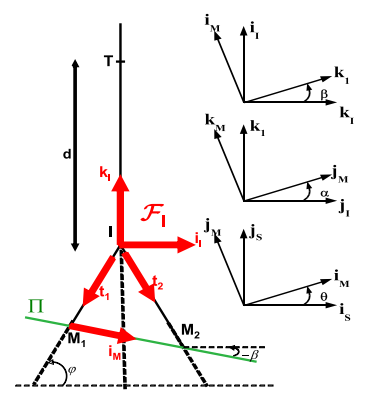

(b) Parameters description
Fig. 5. System Modelling

- $\mathcal{F}_{S}=\left\{S ; \mathbf{i}_{S}, \mathbf{j}_{S}, \mathbf{k}_{S}\right\}$ is the coordinate frame attached to the ultrasound probe $\mathcal{S}$ with $\mathbf{k}_{S}$ perpendicular to the ultrasound plane, and $S$ the origin of the ultrasound rays,

- $\mathcal{F}_{I}=\left\{I ; \mathbf{i}_{I}, \mathbf{j}_{I}, \mathbf{k}_{I}\right\}$ is the coordinate frame attached to the instrument $\mathcal{I}$, with $I$ being the intersection point between the two lines representing the jaws and the line representing the instrument axis. The vector $\mathbf{k}_{I}$ is parallel to the instrument axis, Fig. 5(b).

- $\mathcal{F}_{M}=\left\{M_{1} ; \mathbf{i}_{M}, \mathbf{j}_{M}, \mathbf{k}_{S}\right\}$, where $\mathbf{i}_{M}$ is defined as $\mathbf{i}_{M}=\frac{\mathbf{d}_{\mathbf{M}_{1} \mathbf{M}_{2}}}{\| \mathbf{d}_{\mathbf{M}_{1} \mathbf{M}_{2} \|}}$.

Moreover, we will use in the next the following notations: $\mathbf{d}_{T I}=-d \mathbf{k}_{I}, \mathbf{d}_{I M_{i}}=l_{i} \mathbf{t}_{i}$ for $i=\{1,2\}$, ${ }^{I} \mathbf{t}_{1}=\left[\begin{array}{lll}-t_{x} & 0 & -t_{z}\end{array}\right]^{T}$ and ${ }^{I} \mathbf{t}_{2}=\left[\begin{array}{lll}t_{x} & 0 & -t_{z}\end{array}\right]^{T}$ with $t_{x}^{2}+t_{z}^{2}=1$

\section{Image jacobian}

As the instrument is introduced into the heart through a trocar fixed on the heart wall, only four intracardiac degrees of freedom remain, Fig. 4(b). Therefore, the command sent to the robot can be expressed as:

$$
\mathbf{u}=\left[\omega_{y}, \dot{d}, \omega_{x}, \omega_{z}\right]^{T}
$$

where $\left[\omega_{x}, \omega_{y}, \omega_{z}\right]^{T}={ }^{I} \boldsymbol{\Omega}_{\mathcal{I} / \mathcal{S}}$ is the angular velocity of the instrument tip with respect to the ultrasound probe and $\dot{d}$ is the translation velocity of the instrument tip along $\mathbf{k}_{\mathbf{I}}$. In order to compute for $\dot{\mathbf{s}}$ with respect to $\mathbf{u}$, one first writes $\dot{\mathrm{s}}$ in the base $\mathcal{B}_{\mathcal{M}}$ :

$$
\begin{aligned}
\dot{\mathbf{s}}= & {\left[\begin{array}{c}
{ }^{S} \mathbf{V}\left(\mathbf{M}_{\mathbf{i}} / \mathcal{S}\right)_{\mathbf{x}} \\
\dot{\delta} \\
{ }^{S} \mathbf{V}\left(\mathbf{M}_{\mathbf{i}} / \mathcal{S}\right)_{\mathbf{y}} \\
\dot{\theta}
\end{array}\right] } \\
= & \underbrace{\left[\begin{array}{cccc}
\cos \theta & 0 & -\sin \theta & 0 \\
0 & 1 & 0 & 0 \\
\sin \theta & 0 & \cos \theta & 0 \\
0 & 0 & 0 & 1
\end{array}\right]}_{=\mathbf{R}} \underbrace{\left[\begin{array}{c}
{ }^{M} \mathbf{V}\left(\mathbf{M}_{\mathbf{i}} / \mathcal{S}\right)_{\mathbf{x}} \\
\dot{\delta} \\
\mathbf{V}\left(\mathbf{M}_{\mathbf{i}} / \mathcal{S}\right)_{\mathbf{y}} \\
\dot{\theta}
\end{array}\right]}_{=\mathbf{v}}
\end{aligned}
$$


Thus, the image jacobian $\mathbf{J}_{\mathbf{s}}$ can be decomposed as:

$$
\mathbf{J}_{\mathbf{s}}=\mathbf{R J}
$$

where $\mathbf{J}$ is defined by:

$$
\mathbf{v}=\mathbf{J u}
$$

In the next, each line of $\mathbf{J}$ is computed separately.

1) Velocity of point $P$ : The relation giving the velocity of one point $M_{i}$ in the image as a function of the instrument velocity is, [1]:

$$
\begin{aligned}
\mathbf{V}\left(\mathbf{M}_{\mathbf{i}} / \mathcal{S}\right)= & \frac{-1}{\mathbf{k}_{\mathbf{S}}^{\mathbf{T}} \mathbf{t}_{\mathbf{i}}} \mathbf{k}_{\mathbf{S}} \times \\
& {\left[\mathbf{t}_{\mathbf{i}} \times\left(-\dot{d} \mathbf{k}_{\mathbf{I}}+\left(d \mathbf{k}_{\mathbf{I}}-l_{i} \mathbf{t}_{\mathbf{i}}\right) \times \boldsymbol{\Omega}_{\mathcal{I} / \mathcal{S}}\right)\right] }
\end{aligned}
$$

This relation, expressed in the base $\mathcal{B}_{\mathcal{M}}$, can be rewritten into the following matrix equation:

$$
\left[\begin{array}{l}
{ }^{M} \mathbf{V}\left(\mathbf{M}_{\mathbf{i}} / \mathcal{S}\right)_{\mathbf{x}} \\
{ }^{M} \mathbf{V}\left(\mathbf{M}_{\mathbf{i}} / \mathcal{S}\right)_{\mathbf{y}}
\end{array}\right]=\left[\begin{array}{l}
\mathbf{J}_{\mathbf{M}_{\mathbf{i}} x} \\
\mathbf{J}_{\mathbf{M}_{\mathbf{i}} y}
\end{array}\right] \mathbf{u}
$$

with

$$
\begin{aligned}
& \mathbf{J}_{\mathbf{M}_{\mathbf{i} x}}=\left[\begin{array}{llll}
\frac{l+d t_{z}}{N} & \frac{t_{i x}}{N} & \frac{\left(l t_{z}+d\right) D \tan \alpha}{N} & \frac{l t_{i x} D \tan \alpha}{N}
\end{array}\right] \\
& \mathbf{J}_{\mathbf{M}_{\mathbf{i} y}}=\left[\begin{array}{llll}
0 & 0 & \frac{l t_{z}+d}{\cos \alpha} & \frac{l t_{i x}}{\cos \alpha}
\end{array}\right] \\
& D=t_{i x} \cos \beta+t_{z} \sin \beta \quad N=t_{i x} \sin \beta-t_{z} \cos \beta \\
& t_{1 x}=-\cos \phi \quad t_{2 x}=\cos \phi \quad t_{z}=\sin \phi
\end{aligned}
$$

Thus to obtain the relation giving the velocity of the point $P$, one needs to compute:

$$
\left[\begin{array}{l}
{ }^{M} \mathbf{V}(\mathbf{P} / \mathcal{S})_{\mathbf{x}} \\
{ }^{M} \mathbf{V}(\mathbf{P} / \mathcal{S})_{\mathbf{y}}
\end{array}\right]=\left[\begin{array}{l}
\mathbf{J}_{\mathbf{P} x} \\
\mathbf{J}_{\mathbf{P} y}
\end{array}\right] \mathbf{u}=\frac{1}{2}\left[\begin{array}{l}
\mathbf{J}_{\mathbf{M}_{1}}+\mathbf{J}_{\mathbf{M}_{\mathbf{2}}} \\
\mathbf{J}_{\mathbf{M}_{\mathbf{1}}}+\mathbf{J}_{\mathbf{M}_{\mathbf{2}} y}
\end{array}\right] \mathbf{u}
$$

2) Velocity of the angle $\theta$ : The angular velocity of the tip of the instrument is:

$$
\boldsymbol{\Omega}(\mathcal{I} / \mathcal{S})=\boldsymbol{\Omega}(\mathcal{I} / \mathcal{M})+\boldsymbol{\Omega}(\mathcal{M} / \mathcal{S})
$$

Using the angle definition as shown in Fig. 5(b), (12) can be re-written as:

$$
\omega_{x} \mathbf{i}_{\mathbf{I}}+\omega_{y} \mathbf{j}_{\mathbf{I}}+\omega_{z} \mathbf{k}_{\mathbf{I}}=-\dot{\beta} \mathbf{j}_{\mathbf{I}}-\dot{\alpha} \mathbf{j}_{\mathbf{M}}+\dot{\theta} \mathbf{k}_{\mathbf{S}}
$$

To identify the terms leads to:

$$
\dot{\theta}=\mathbf{J}_{\theta} \mathbf{u}=\left[\begin{array}{llll}
0 & 0 & \frac{\sin \beta}{\cos \beta} & \frac{\cos \beta}{\cos \alpha}
\end{array}\right] \mathbf{u}
$$

3) Velocity of the distance $\delta$ : The distance between $M_{1}$ and $M_{2}$ is:

$$
\delta=\left\|\mathbf{M}_{\mathbf{1}} \mathbf{M}_{\mathbf{2}}\right\|=\mathbf{M}_{\mathbf{1}} \mathbf{M}_{\mathbf{2}}^{T} \cdot \mathbf{i}_{\mathbf{M}}
$$

Its velocity is then given by:

$$
\dot{\delta}=\left(\mathbf{V}\left(\mathbf{M}_{\mathbf{2}} / \mathcal{S}\right)-\mathbf{V}\left(\mathbf{M}_{\mathbf{1}} / \mathcal{S}\right)\right)^{T} ._{\mathbf{M}}
$$

Therefore:

$$
\dot{\delta}=\mathbf{J}_{\delta} \mathbf{u}=\left[\mathbf{J}_{\mathbf{M}_{2 x}}-\mathbf{J}_{\mathbf{M}_{1 x}}\right] \mathbf{u}
$$

4) Final kinematic model: Finally, the kinematic model of the system can be written as:

$$
\mathbf{v}=\mathbf{J u}=\left[\begin{array}{c}
\mathbf{J}_{\mathbf{P} x} \\
\mathbf{J}_{\delta} \\
\mathbf{J}_{\mathbf{P} y} \\
\mathbf{J}_{\theta}
\end{array}\right] \mathbf{u}
$$

Note that the jacobian matrix is a block triangular matrix (which a posteriori justifies the choice for ordering the coordinates of both $\mathbf{s}$ and $\mathbf{u}$ ). This means that the two commands $\omega_{x}$ and $\dot{d}$ will produce neither velocity of point $P$ along $\mathbf{j}_{\mathbf{M}}$ nor rotation of the instrument in the image. This block-diagonal structure is a nice property of the proposed parameterization, as it provides a significant increase of the visual servoing loop robustness [13].

5) Image jacobian estimation: It can be noticed that, if the angles $\alpha$ and $\beta$ are both equal to zero, then the jacobian matrix becomes diagonal and writes:

$$
\mathbf{J}_{(\mathbf{0}, \mathbf{0})}=\left[\begin{array}{cccc}
-\frac{\sin (2 \phi) d+\delta}{\sin (2 \phi)} & 0 & 0 & 0 \\
0 & \frac{-2}{\tan \phi} & 0 & 0 \\
0 & 0 & \frac{2 d+\tan \phi}{2} & 0 \\
0 & 0 & 0 & 1
\end{array}\right]
$$

In the next, it will be supposed that the geometric configuration of the system is chosen such that the ultrasound plane is approximately perpendicular to the instrument axis. In this case, the matrix $\mathbf{J}$ is approximately diagonal, and its estimate will be set as:

$$
\widehat{\mathbf{J}_{\mathbf{s}}}=\mathbf{R} \mathbf{J}_{(\mathbf{0}, 0)}
$$

Note that $\mathbf{R}$, which only depends on the measured parameter $\theta$ can be assumed to be perfectly known. From (1), the control law is finally:

$$
\mathbf{u}=-\lambda \widehat{\mathbf{J}_{s}^{-1}} \boldsymbol{\varepsilon}=-\lambda \widehat{\mathbf{J}^{-1}} \mathbf{R}^{T} \boldsymbol{\varepsilon}
$$

A very interesting feature of this control law is that it does not require any on-line estimation of the probe location with respect to the robot. This feature is quite interesting in the context of a medical application, as it allows for not using, in the system, any additional external localizer for registration.

\section{Simulation Results}

Numerical simulations were performed to evaluate the closed loop behavior of the system. In Fig. 6, the simulation is performed with $\lambda=1$, considering a case where the instrument is perpendicular (at the desired configuration) to the ultrasound plane, with a small displacement involved. In this case, one has $\widehat{\mathbf{J}_{\mathbf{s}}} \approx \mathbf{J}_{\mathbf{s}}$, so that (3) can be re-written as:

$$
\dot{\varepsilon}=-\lambda \mathbf{J}_{\mathbf{s}} \widehat{\mathbf{J}_{\mathbf{s}}^{-1}} \varepsilon \approx-\lambda \mathbf{J}_{\mathbf{s}} \mathbf{J}_{\mathbf{s}}^{-1} \varepsilon=-\varepsilon
$$

This equation is linear for $\varepsilon$ and the error exponentially converges toward zero.

Moreover, additional simulations indicate that even with large orientations between the instrument and the perpendicular 

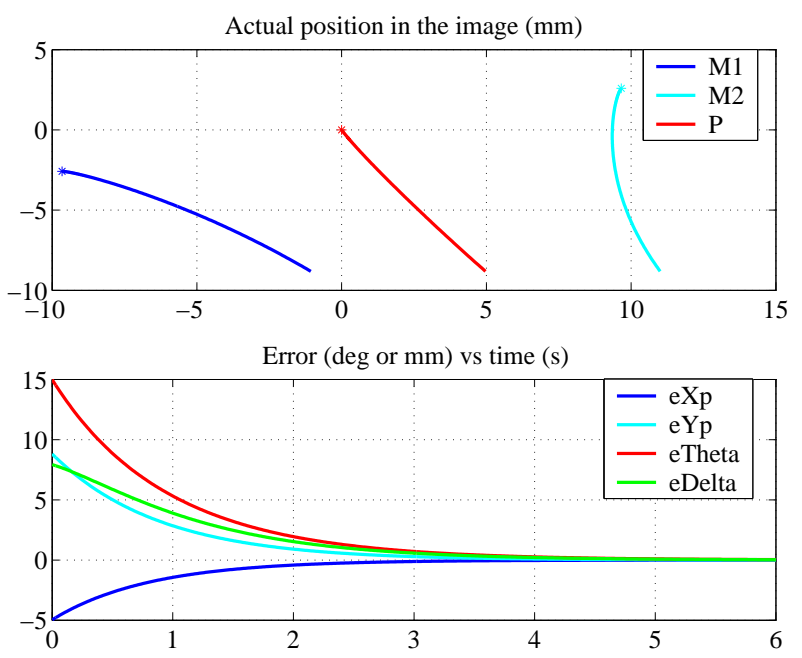

Fig. 6. Simulation results in the perpendicular case

to the probe plane, the error still converges toward zero and the control loop is still stable. As an illustration, Fig. 7 shows simulation results when the orientation reaches $\alpha=50$ degrees and $\beta=35$ degrees. In spite of this large orientation, the system is still stable.
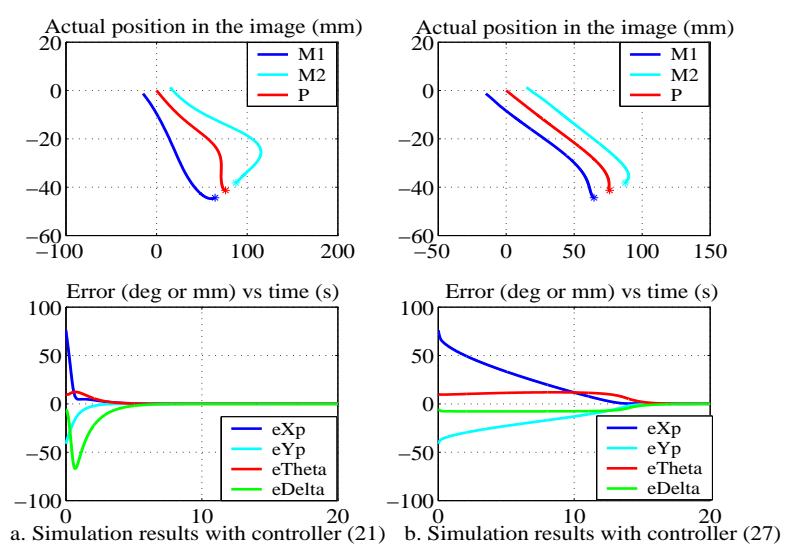

Fig. 7. Simulation results with large orientation

Although the stability is maintained, one can see in Fig. 7.a that the distance $\delta$ between the two blobs reaches approximately $8 \mathrm{~cm}$. This distance is not realistic as the jaws length is, in practice, limited, which was not accounted for in the simulation. In fact, in a real situation, the jaws would not intersect the plane anymore, which means that the controller would fail. This corresponds to the well known visibility constraint in conventional visual servoing. To overcome this problem, we have designed a modified control law, that allows for a better control of the distance $\delta$, and, in fine, a better guarantee for the instrument visibility.

\section{Visual SERVOING With ENHANCED DEPTH CONTROL}

With the control law (21), the closed loop behavior for the error in $\delta$ is

$$
\dot{\varepsilon}_{\delta}=\mathbf{J}_{\delta} \mathbf{u}=\mathbf{J}_{\delta}\left[\begin{array}{l}
-\lambda \widehat{\mathbf{J}_{11}^{-1}} \varepsilon_{x}^{\prime} \\
-\lambda \widehat{\mathbf{J}_{22}^{-1}} \varepsilon_{\delta} \\
-\lambda \widehat{\mathbf{J}_{33}^{-1}} \varepsilon_{y}^{\prime} \\
-\lambda \widehat{\mathbf{J}_{44}^{-1}} \varepsilon_{\theta}
\end{array}\right]
$$

where $\varepsilon^{\prime}=\mathbf{R} \varepsilon$. It can be seen that large errors on $\varepsilon_{x}^{\prime}, \varepsilon_{y}^{\prime}$ or $\varepsilon_{\theta}$ can induce large velocities for $\dot{\varepsilon}_{\delta}$. This is due to the fact that the control law does not perfectly decouple the system. As a result, even when $\varepsilon_{\delta}$ is (e.g.) positive, the coupling terms can result in a positive value for $\dot{\varepsilon}_{\delta}$, which leads to large errors. To cope with this problem, we wish to bound $\varepsilon_{\delta}$ by:

$$
-\varepsilon_{\delta \max }<\varepsilon_{\delta}<\varepsilon_{\delta \max }
$$

In other words, we want that:

- if $\varepsilon_{\delta} \geq \varepsilon_{\delta \max }$ then $\dot{\varepsilon}_{\delta}<0$

- if $\varepsilon_{\delta} \leq-\varepsilon_{\delta \max }$ then $\dot{\varepsilon}_{\delta}>0$

A simple control law that provides this property is:

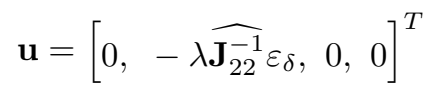

Indeed, the closed loop behavior for $\varepsilon_{\delta}$ is then:

$$
\dot{\varepsilon}_{\delta}=-\lambda \mathbf{J}_{\delta_{2}} \widehat{\mathbf{J}_{22}^{-1}} \varepsilon_{\delta}
$$

and $\mathbf{J}_{\delta_{2}}$ and $\widehat{\mathbf{J}_{22}^{-1}}$ have the same sign. However, obviously, the control law (25) does not allow for the three other coordinates of $\mathbf{s}$ to converge. Therefore, the idea is to switch between from control law (21) to control law (25) when the error $\varepsilon_{\delta}$ reaches its bound. In order to avoid for chattering induced by switches between control laws, a continuous controller is implemented, which final form is:

$$
\mathbf{u}=\left[-\rho \widehat{\mathbf{J}_{11}^{-1}} \varepsilon_{x}^{\prime}, \quad-\lambda \widehat{\mathbf{J}_{22}^{-1}} \varepsilon_{\delta},-\rho \widehat{\mathbf{J}_{33}^{-1}} \varepsilon_{y}^{\prime}, \quad-\rho \widehat{\mathbf{J}_{44}^{-1}} \varepsilon_{\theta}\right]
$$

where:

$$
\rho= \begin{cases}\lambda & \text { if }\left|\varepsilon_{\delta}\right|<\varepsilon_{0} \\ \lambda \frac{\varepsilon_{\delta \max }-\varepsilon}{\varepsilon_{\delta \max }-\varepsilon_{0}} & \text { if } \varepsilon_{0} \leq\left|\varepsilon_{\delta}\right| \leq \varepsilon_{\delta \max } \\ 0 & \text { otherwise }\end{cases}
$$

This control law corresponds to controller (21) if $\left|\varepsilon_{\delta}\right|<\varepsilon_{0}$, controller (25) if $\left|\varepsilon_{\delta}\right|>\varepsilon_{\delta \max }$, and a linear interpolation between the two controllers when $\varepsilon_{0} \leq\left|\varepsilon_{\delta}\right| \leq \varepsilon_{\delta \text { max }}$. Figure Fig. 7.b illustrates the efficiency of the approach. Indeed, it can be seen that the distance $\delta$ is well controlled which ensured the instrument visibility along the path.

\section{IN VIVO EXPERIMENT}

In vivo experiments have been performed on pig at the surgical school of Paris, Fig. 8(a). In these experiments, the ultrasound probe used is manually maintained in contact with the heart as transoesophagus probe cannot be used on porks. The robot $(\mathrm{MC} 2 \mathrm{E}$, french acronym for compact manipulator 


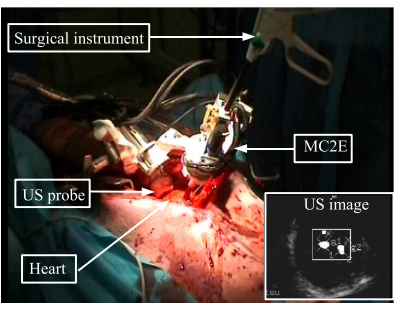

(a) set-up

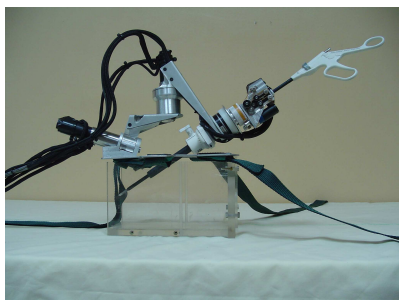

(b) $\mathrm{MC} 2 \mathrm{E}$
Fig. 8. In vivo set-up

for endoscopic surgery, developed at the Laboratoire de Robotique de Paris) used to manipulate the instrument is especially suited for minimally invasive robotic surgery applications and provides, with its spherical structure, 4 degrees of freedom at the instrument tip, Fig. 8(b).The design of the robot is presented in [14].

The experiment is performed as follows:

The user specified the desired goal in the ultrasound image. Then the current instrument coordinates in the image and the corresponding error are calculated and transmitted via a TCP/IP connection to the visual servoing loop. Thereafter, the command velocity of the instrument is computed according to (1) and sent to the robot controller.
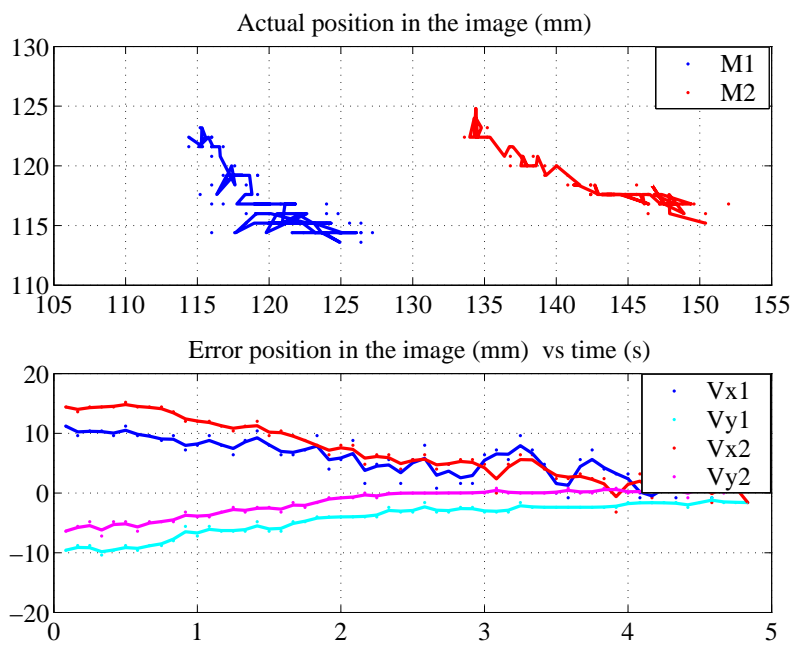

Fig. 9. In vivo experiment results

Figure 9 shows the in vivo experiment results. These results are noisy because of numerous artifacts in the ultrasound images. Eventhough, the error converges toward zero and the desired position is reached.

\section{CONCLUSION}

A robotic system with ultrasound imaging is presented. This system uses ultrasound based visual servoing to control the position of an instrument inside the heart. Modeling of the system and control loop are developed. Remarkably, the controller does not require any explicit model for the location of the probe with respect to the robot. Simulations predict good robustness of the visual servoing loop. In vivo experiments were successfully performed.

\section{ACKNOWLEDGMENTS}

The financial support of the CNRS P.I.R. Robea program (project GABIE) is gratefully acknowledged. Thanks to the partners of this project: LIRMM, Montpellier, TIMC, Grenoble, CEA-FAR, CHUG, Grenoble, Pitié Salpêtrière, Paris.

\section{REFERENCES}

[1] M.-A. Vitrani, G. Morel, and T. Ortmaier. Automatic guidance of a surgical instrument with ulatrasound based visual servoing. In Proc. of the ICRA 2005, Barcelona - Spain, April 2005.

[2] L. Angelini and M. Caratozzolo. Intraoperative echography: the state of the art. Ann. Ital. Chir, 70(2):223-230, March-April 1999.

[3] A. Gourdon, P. Poignet, G. Poisson, Y. Parmantier, and P. Marche. Master slave robotic system for ultrasound scanning. In IEEE Medical and Biological Engineering Conference, Proceedings, Vienna, Austria, November 1999.

[4] A. Vilchis, J. Troccaz, P. Cinquin, A. Guerraz, F. Pellisier, P. Thorel, B. Tondu, F. Courrges, G. Poisson, M. Althuser, and J. Ayoubi. Experiments with the ter tele-echography robot. In Medical Image Computing and Computer-Assisted Intervention - MICCAI 2002: 5th International Conference, Proceedings, Part I, pages 138 - 146, Tokyo, Japan, September 25-28 2002.

[5] F. Pierrot, E. Dombre, E. Dégoulange, L. Urbain, P. Caron, S. Boudet, J. Gariépy, and J.L. Mégnien. Hippocrate: a safe robot arm for medical applications with force feedback. Medical Image Analysis, 3(3):285300, 1999.

[6] S.E. Salcudean, W.H. Zhu, P. Abolmaesumi, S. Bachmann, and P.D. Lawrence. A robot system for medical ultrasound. Robotics Research, May 2000.

[7] A. Krupa and F. Chaumette. Control of an ultrasound probe by adaptive visual servoing. In IEEE/RSJ Int. Conf. on Intelligent Robots and Systems, IROS'05, volume 2, pages 2007-2012, Edmonton, Canada, August 2005.

[8] G. Megali, O. Tonet, C. Stefanini, M. Boccadoro, V. Papaspyropoulos, L. Angelini, and P. Dario. A computer-assisted robotic ultrasoundguided biopsy system for video-assisted surgery. In Medical Image Computing and Computer-Assisted Intervention - MICCAI 2001: 4th International Conference, Proceedings, pages 343-350, Utrecht, The Netherlands, October 2001.

[9] J. Stoll, P. Dupont, and R. Howe. Ultrasound-based servoing of manipulators for telesurgery. In Telemanipulator and Telepresence Technologies VIII Conference, volume 4570 of Proceedings of SPIE, October 2001.

[10] J. Hong, T. Dohi, M. Hashizume, K. Konishi, and N. Hata. An ultrasound-driven needle insertion robot for percutaneous cholecystostomy. Physics in Medicine and Biology, 49(3):441-455, february 2004.

[11] T. Ortmaier, M.-A. Vitrani, G. Morel, and S. Pinault. Robust real-time instrument tracking in ultrasound images. In Proc. of SPIE Medical Imaging Conference, San Diego, California, USA, February 2005.

[12] Gregory Hager, Seth Hutchinson, and Peter Corke. Tutorial TT3: A tutorial on visual servo control. Held at ICRA 1994 Conference.

[13] E. Malis and F. Chaumette. Theoretical improvements in the stability analysis of a new class of model-free visual servoing methods. IEEE Transaction on Robotics and Automation, 18(2):176-186, April 2002.

[14] N. Zemiti, T. Ortmaier, M.-A. Vitrani, and G. Morel. A force controlled laparoscopic surgical robot without distal force sensing. In Proc. of the ISER 2004; 9th International Symposium on Experimental Robotics, Singapore, June 2004. 